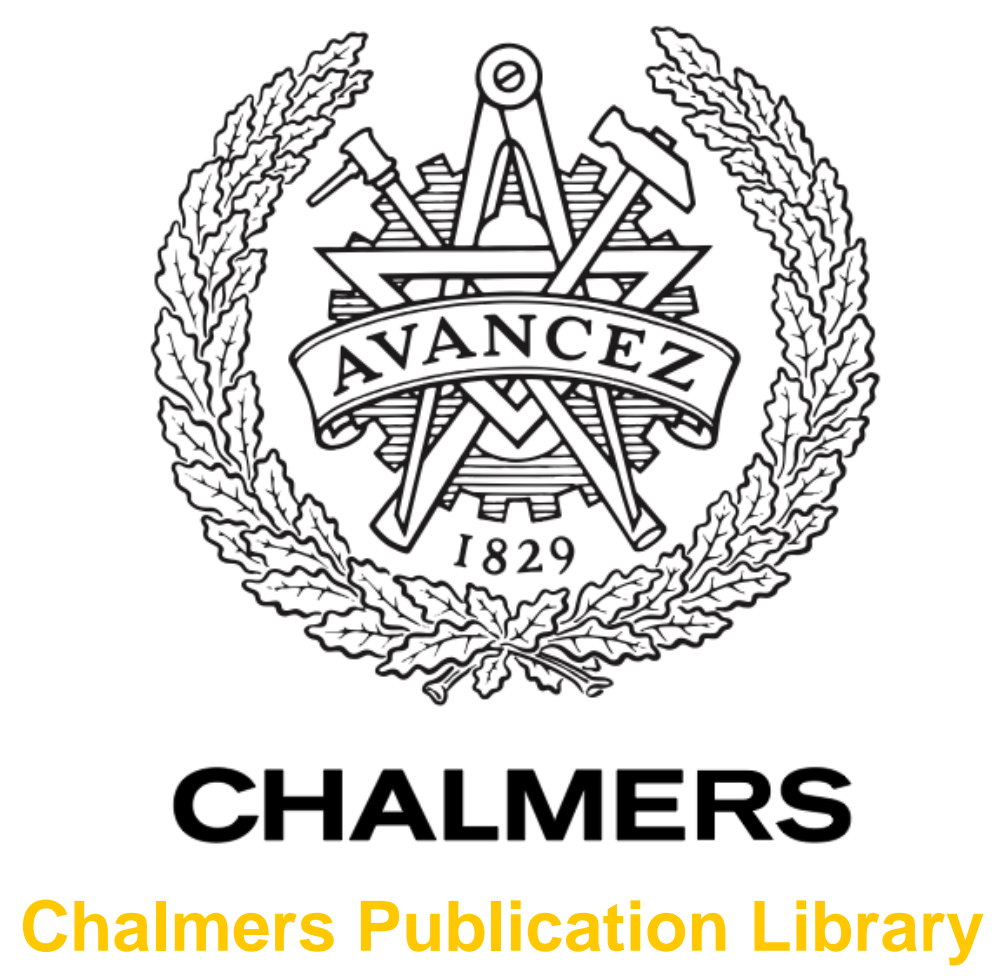

Extending Digital Backpropagation to Account for Noise

This document has been downloaded from Chalmers Publication Library (CPL). It is the author's version of a work that was accepted for publication in:

Proc. of 39th European Conference and Exhibition on Optical Communication (ECOC 2013)

Citation for the published paper:

Irukulapati, N. ; Wymeersch, H. ; Johannisson, P. (2013) "Extending Digital

Backpropagation to Account for Noise". Proc. of 39th European Conference and Exhibition on Optical Communication (ECOC 2013) pp. We.3.C.4.

Downloaded from: http://publications.lib.chalmers.se/publication/186523

Notice: Changes introduced as a result of publishing processes such as copy-editing and formatting may not be reflected in this document. For a definitive version of this work, please refer to the published source. Please note that access to the published version might require a subscription.

Chalmers Publication Library (CPL) offers the possibility of retrieving research publications produced at Chalmers University of Technology. It covers all types of publications: articles, dissertations, licentiate theses, masters theses, conference papers, reports etc. Since 2006 it is the official tool for Chalmers official publication statistics. To ensure that Chalmers research results are disseminated as widely as possible, an Open Access Policy has been adopted.

The CPL service is administrated and maintained by Chalmers Library. 


\title{
Extending Digital Backpropagation to Account for Noise
}

\author{
Naga V. Irukulapati(1), Henk Wymeersch ${ }^{(1)}$, Pontus Johannisson ${ }^{(2)}$, and Erik Agrell(1)
}

(1) Department of Signals and Systems, (2) Department of Microtechnology and Nanoscience, Chalmers University of Technology, Gothenburg, SE-41296, Sweden. $\bowtie$ vnaga@chalmers.se

\begin{abstract}
We propose a maximum a posteriori-based scheme that extends digital backpropagation (DBP) by accounting for the nonlinear signal-noise interaction. With periodic dispersion compensation we find up to $20 \%$ reach improvement over DBP. For uncompensated links DBP is close to optimal.
\end{abstract}

\section{Introduction}

The propagation of light in an optical fiber can be modeled by the Manakov model with loss included. The Manakov model does not generally have an analytic solution and numerical approaches, such as the split-step Fourier method (SSFM), are often used to describe the signal propagation in dispersive and nonlinear media ${ }^{1}$. Since a fiber is a lossy medium, periodic amplification of the signal is needed, which can be accounted for in the SSFM. However, amplifiers also add noise and the signal-noise interaction due to nonlinear effects must be modeled statistically.

Digital backpropagation (DBP), which is often used as a benchmark algorithm, harnesses the invertible nature of the linear and nonlinear impairments to derive a digital receiver that inverts the $\mathrm{SSFM}^{2,3}$. Variations include weighted $\mathrm{DBP}^{4}$, perturbation $\mathrm{DBP}^{5}$, and filtered $\mathrm{DBP}^{6}$, to name a few. However, all DBP variations fail to capture the statistical effects of the channel, in particular the noise introduced in the amplifiers, and are thus suboptimal. Receivers can also be designed from Bayesian detection theory. In particular, the maximum a posteriori (MAP) receiver is provably optimal in the sense of minimizing the probability of error ${ }^{7}$. MAP-based detectors for the fiberoptical channels include a look-up-table detector that mitigates data pattern-dependent nonlinear impairments ${ }^{8,9}$, a low-complexity Viterbi detector that complements $\mathrm{DBP}^{10}$, and an optimal detector for discrete memoryless channels ${ }^{11}$.

In this paper, we extend the MAP-based detector for the single-channel ${ }^{11}$ to account for dispersive effects. The proposed detector is based on the MAP criterion and compensates not only linear and nonlinear effects but also takes the noise from the amplifiers into account. As a consequence, nonlinear signalnoise interactions (NSNI) can be handled using the proposed detector. This allows us to (i) get closer to the fundamental performance limits of the fiberoptical channel; and (ii) identify regimes where DBP is close to optimal. Our proposed near-MAP detector turns out to be a generalization of DBP, and hence we call the method stochastic digital backpropagation $(S D B P)$.

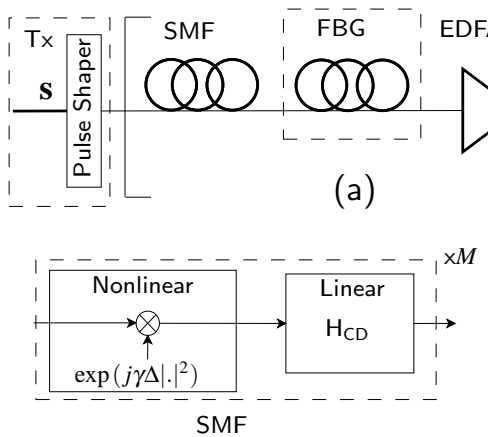

(b)

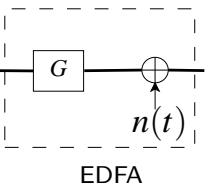

(c)
Fig. 1: (a) A fiber link with $N$ spans where each span consists of an SMF, an FBG (for the compensated case), and an EDFA. (b) Approximate model for SMF using SSFM with nonlinear and linear segments. (c) EDFA with gain and noise.

\section{System Model}

Notation: The vector representation corresponding to the dual-polarization continuous-time waveform $\mathbf{q}(t)$ will be denoted by $\mathbf{q}$, and vector representation of a collection of waveforms by $\mathbf{Q}$.

The system model is shown in Fig. 1a, and comprises a dual-polarization transmitter, a pulse shaper, and $N$ spans of fiber, each consisting of a standard single-mode fiber (SMF) followed by an optional fiber Bragg grating (FBG) for the case of inline dispersion compensation and an erbium-doped fiber amplifier (EDFA). In Fig. 1b, we show the SSFM view of the system, where each SMF is approximated by the concatenation of $M$ nonlinear memoryless operators (Kerr nonlinearity) and linear operators (chromatic dispersion). Fig. 1c shows the operation of the amplifier, consisting of gain and addition of white Gaussian noise (AWGN) with power spectral density (PSD) $N_{0} / 2$ per real dimension. A sequence of $K$ transmitted complex symbols per polarization is denoted by $\mathbf{s} \in \Omega^{(2 K)}$ (for a complex constellation $\Omega$ ), the transmitted waveform by $\mathbf{x}$, and the received waveform by $\mathbf{r}$. In Fig. 1, the channel with SMF, FBG (for compensated links only), and EDFA will be called a forward system. When the received waveform is propagated through the inverse of the blocks in the forward system, we refer to it as a backward system. 


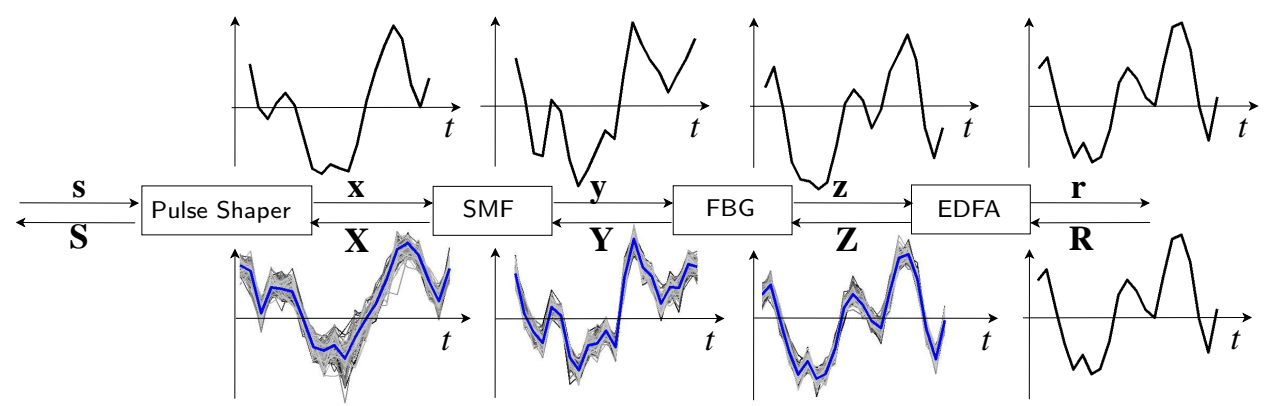

Fig. 2: A simplified system model with unknown symbol sequence $\mathbf{s}$ passing through different blocks of the channel with $N=1$ spans, and received waveform $\mathbf{r}$. The top row depicts the waveforms corresponding to the hidden states $\mathbf{X}, \mathbf{y}, \mathbf{Z}$ as well as the received waveform $\mathbf{r}$. In the bottom row, collections of waveforms representing the uncertainty of the hidden states are shown in gray and the DBP waveform is shown in thick blue lines.

\section{Proposed MAP-based Detector}

Formally, the MAP detector calculates $\hat{\mathbf{s}}=$ $\arg \max _{\mathbf{s}} p(\mathbf{s} \mid \mathbf{r})$, where $p(\mathbf{s} \mid \mathbf{r})$ is the posterior distribution of $\mathbf{s}$. The posterior can be interpreted as a marginal distribution of $p(\mathbf{s}$, hidden states $\mid \mathbf{r})$, where the hidden states refer to the unobservable waveforms between the transmitter and the receiver (e.g., the waveform after an arbitrarily chosen SMF or FBG or EDFA). Fig. 2 is a simplified case of Fig. 1 for $N=1$, where we have the transmitted data $\mathbf{s}$, hidden states $\mathbf{x}, \mathbf{y}, \mathbf{z}$, and the received waveform $\mathbf{r}$. The top row of Fig. 2 depicts the hidden waveforms as well as the received waveform $\mathbf{r}$ in the forward system.

We propose an algorithm based on factor graphs and the sum-product algorithm ${ }^{7,12}$ to approximate $p(\mathbf{s} \mid \mathbf{r})$ and thus perform near-MAP detection. The main idea is to represent the uncertainty in the hidden states at each stage in the forward system, starting from the received waveform $\mathbf{r}$, which has no uncertainty, to the first hidden state ( $\mathbf{z}$ in Fig. 2), all the way to the transmitted sequence $\mathbf{s}$. Uncertainty is captured using a so-called particle representation though a collection of $N_{p}$ sampled waveforms, as shown in gray color in the bottom row of Fig. 2. These particles are passed through the inverse of each of the blocks in the forward system. For example (see Fig. 2), we start with the known received waveform $\mathbf{r}$ (which exhibits no uncertainty and thus has particle representation $\mathbf{R}=\mathbf{r}$ ) and pass it through the inverse of the EDFA block to get $\mathbf{Z}$, a collection of $N_{p}$ waveforms, which describes the uncertainty regarding the waveform $\mathbf{z}$. Since the EDFA block adds AWGN noise with PSD $N_{0} / 2$ per dimension, the waveforms in $\mathbf{Z}$ are generated from $\mathbf{r}$ by adding suitable AWGN processes. We then move on to the next block and undo the effects of the FBG to get the uncertainty of the hidden waveform $\mathbf{y}$ represented by $\mathbf{Y}$. The particles of $\mathbf{X}$ are then found by passing the particles in $\mathbf{Y}$ through the inverse SSFM description of the SMF. It should be noted that even though the SMF block in Fig. 2 is depicted as one block, it contains hidden states corresponding to the SMF internal states as in Fig. 1b, and thus the particles should be passed through each of these internal sub-blocks. Finally, we apply a filter matched to the pulse shape and a symbol-rate sampler. We end up with particle clouds ( $N_{p}$ particles for each complex dimension) corresponding to each symbol vector $\mathbf{s}_{k} \in \Omega^{2}$, serving as a representation of $p\left(\mathbf{r} \mid \mathbf{s}_{k}\right)$ for $k=1,2, \ldots, K$. This likelihood function is approximated with a bivariate complex Gaussian distribution, which is then evaluated for every possible value of $\mathbf{s}_{k}$ and multiplied with $p\left(\mathbf{s}_{k}\right)$. This allows us to make a near-MAP decision on $\mathbf{s}_{k}$ as $\hat{\mathbf{s}}_{k}=\arg \max _{\mathbf{s}} p\left(\mathbf{s}_{k} \mid \mathbf{r}\right)=\arg \max _{\mathbf{s}} p\left(\mathbf{s}_{k}\right) p\left(\mathbf{r} \mid \mathbf{s}_{k}\right)$.

\section{Connection to DBP}

In DBP, once the received waveform $\mathbf{r}$ is available, it is passed through the inverse of each of the blocks used in the forward system and the most likely symbol is selected based on a minimum Euclidean distance criterion. The DBP waveforms are shown as thick blue lines in the bottom row of Fig. 2. We note that the DBP waveform corresponds to a special case of SDBP where (i) $N_{p}=1$ and (ii) the noise in the forward system is ignored.

\section{Simulations and Discussion}

We applied DBP and the proposed detector with $N_{p}=500$ to a system with SMF with dispersion coefficient $D_{\mathrm{SMF}}=16 \mathrm{ps} /(\mathrm{nm} \mathrm{km})$, Kerr nonlinearity parameter $\gamma_{S M F}=1.3 \mathrm{~W}^{-1} \mathrm{~km}^{-1}$, attenuation $\alpha_{\mathrm{SMF}}=0.2 \mathrm{~dB} / \mathrm{km}$, and span length $L_{\mathrm{SMF}}=80 \mathrm{~km}$. For the case with inline dispersion compensation, we used an FBG without Kerr nonlinearity, insertion loss of $3 \mathrm{~dB}$, and perfect dispersion compensation of the preceding SMF. The EDFA noise figure is $5 \mathrm{~dB}$. We used a root raised cosine pulse in time domain with a roll-off factor of 0.25 and truncation length of 16 symbols, and dual polarization 16-QAM. The receiver is assumed to have perfect knowledge of the polarization state, as well as the carrier phase and the symbol timing. ASE noise with bandwidth equal to the used sampling frequency, which is twice the signal bandwidth, is added in each span. The symbol error rate (SER) is used as a performance metric.

The SSFM is simulated with a segment length ${ }^{13}$ of $\Delta=\left(\varepsilon L_{\mathrm{N}} L_{\mathrm{D}}^{2}\right)^{1 / 3}$, where $\varepsilon=10^{-4}, L_{\mathrm{N}}=1 /\left(\gamma_{\mathrm{SMF}} P\right)$ is the nonlinear length, $L_{\mathrm{D}}=T^{2} /\left|\beta_{2}\right|$ is the dispersion length, $P$ is the input power to each fiber span, $T$ is the symbol duration, and $\beta_{2}$ is the group-velocity dispersion parameter of the SMF. The number of 


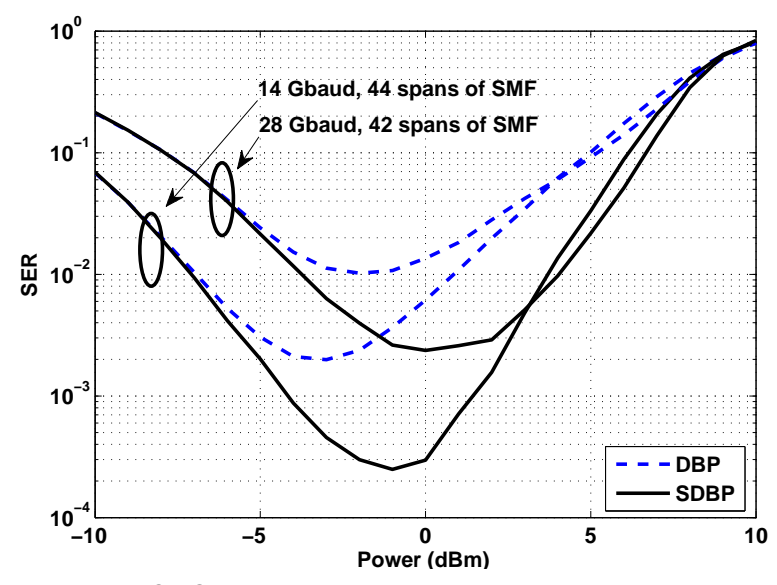

Fig. 3: SER vs. input power for the FBG link.

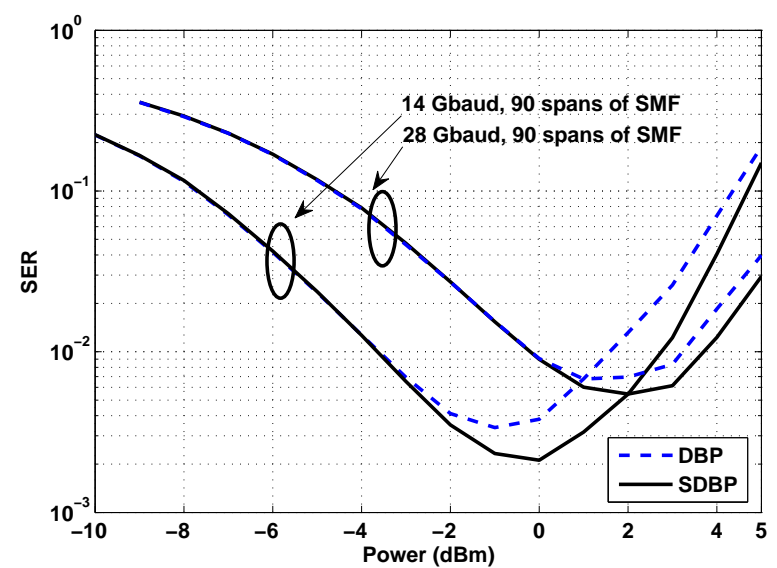

Fig. 4: SER vs. input power for the uncompensated link.

segments per span $M=\left\lceil L_{\mathrm{SMF}} / \Delta\right\rceil$, where $\lceil p\rceil$ is the smallest integer not less than $p$. We used the same segment length in the backward and forward system.

Fig. 3 shows the SER as a function of the input power for two different symbol rates (14 and 28 Gbaud) for a link with FBG dispersion management (called FBG link from here on) for DBP and SDBP. We observe that SDBP performs significantly better than DBP for both symbol rates. Using SDBP, a different optimal power is obtained and also for a given input power, lower SER is obtained. Using SDBP, the system is more tolerant to nonlinear effects and therefore we can use higher launch power. In Fig. 4, we show the SER for a system without inline dispersion compensation. We see that for both symbol rates, SDBP outperforms DBP to some extent, and the gains are smaller for the higher symbol rate. This indicates that DBP is close to optimal for highrate systems without inline dispersion compensation. The results presented in this paper corroborate the result ${ }^{14}$ by quantifying the gains in handling NSNI for both dispersion compensated and uncompensated links. In Fig. 5, we compare the SER against the system reach. Each point in this plot is for an optimal input power, i.e., for different configurations, the input power corresponding to the lowest SER is selected. For 14 Gbaud, we see that there is around $20 \%$ increase in the system reach for SDBP com-

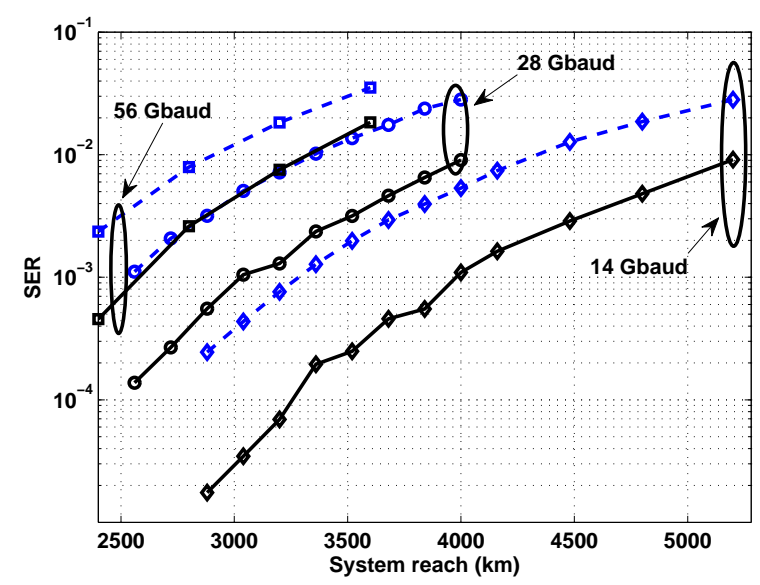

Fig. 5: SER vs. system reach for DBP (dashed, blue) and SDBP (solid, black) for the FBG link.

pared to DBP. However, this gain decreases slightly as we go to higher symbol rates (around 18\% for 28 Gbaud, and $13 \%$ for 56 Gbaud).

Gains of SDBP can be explained as follows. The larger the deviation of the received particle clouds from a circular symmetric Gaussian, the higher are the expected gains in SDBP compared to DBP. We expect this deviation (and thus the gain) to decrease with increasing symbol rates, which can also be seen in Fig. 3. For an inline dispersion compensated link, we observed that the particle clouds are less circularly Gaussian and hence SDBP performs better than DBP. For an uncompensated link, the particle clouds tend to be more circularly Gaussian, which can be the reason why SDBP performs similar to DBP.

\section{Conclusions}

We showed that by taking signal statistics of the end-to-end fiber channel into account, a near-MAP detector can outperform DBP. Our proposed SDBP algorithm shows up to $20 \%$ increase in the system reach compared to DBP for an FBG link. Similar performance for SDBP and DBP for uncompensated links suggests that DBP is then close to optimal.

\section{References}

1 G. P. Agrawal, Nonlinear fiber optics, Academic Press (2007).

2 X. Li et al., Opt. Express, 16, 880 (2008).

3 E. Ip et al., J. Lightw. Technol., 4, 3416 (2010).

4 D. Rafique et al., Opt. Exp., 19, 9453 (2011).

5 T. Hoshida et al., Proc. OFC, OTh3C.3 (2013).

6 L. Du et al., Opt. Exp. 18, 17075 (2010).

$7 \mathrm{H}$. Wymeersch, Iterative Receiver Design, Cambridge University Press (2007).

8 Y. Cai et al., Photonics Society Summer Topical Meeting Series, 42(2010).

9 T. Koike-Akino et al., Opt. Exp., 20, 15769 (2012).

10 D. Marsella et al., Proc. ECOC, P4.06 (2012).

11 N. Jiang et al., Proc. ECOC, We.10.P1.81 (2011).

12 F. R. Kschischang et al., IEEE Trans. Inf. Theory, 2, 498 (2001).

13 Q. Zhang et al., J. Lightw. Technol., 2, 302 (2008).

14 D. G. Foursa et al., Proc. OFC, JTh2A.35, (2013). 